\title{
BMJ Open Comparative efficacy and tolerability of first-generation and newer-generation antidepressant medications for depressive disorders in children and adolescents: study protocol for a systematic review and network meta-analysis
}

\author{
Xinyu Zhou, ${ }^{1}$ Bin Qin, ${ }^{1}$ Craig Whittington, ${ }^{2}$ David Cohen, ${ }^{3}$ Yiyun Liu, ${ }^{1}$ \\ Cinzia Del Giovane, ${ }^{4}$ Kurt D Michael, ${ }^{5}$ Yuqing Zhang, ${ }^{1}$ Peng Xie ${ }^{1}$
}

To cite: Zhou X, Qin B, Whittington $\mathrm{C}$, et al. Comparative efficacy and tolerability of first-generation and newer-generation antidepressant medications for depressive disorders in children and adolescents: study protocol for a systematic review and network meta-analysis. BMJ Open 2015;5:e007768. doi:10.1136/bmjopen-2015007768

- Prepublication history for this paper is available online. To view these files please visit the journal online (http://dx.doi.org/10.1136/ bmjopen-2015-007768).

$\mathrm{XZ}$ and $\mathrm{BQ}$ contributed equally.

Received 23 January 2015 Revised 6 August 2015 Accepted 12 August 2015

\section{CrossMark}

For numbered affiliations see end of article.

Correspondence to Professor Peng Xie; xiepeng973@126.com

\section{ABSTRACT}

Introduction: Depressive disorders are among the most common psychiatric disorders in children and adolescents, and have adverse effects on their psychosocial functioning. Questions concerning the efficacy and safety of antidepressant medications in the treatment of depression in children and adolescents, led us to integrate the direct and indirect evidence using network meta-analysis to create hierarchies of these drugs.

Methods and analysis: Seven databases with PubMed, EMBASE, the Cochrane Library, Web of Science, CINAHL, LiLACS and PsycINFO will be searched from 1966 to December 2013 (updated to May, 2015). There are no restrictions on language or type of publication. Randomised clinical trials assessing first-generation and newer-generation antidepressant medications against active comparator or placebo as acute treatment for depressive disorders in children and adolescents (under 18 years of age) will be included. The primary outcome for efficacy will be mean improvement in depressive symptoms, as measured by the mean change score of a depression rating scale from baseline to post-treatment. The tolerability of treatment will be defined as side effect discontinuation, as defined by the proportion of patients who discontinued treatment due to adverse events during the trial. We will also assess the secondary outcome for efficacy (response rate), acceptability (all-cause discontinuation) and suiciderelated outcomes. We will perform the Bayesian network meta-analyses for all relative outcome measures. Subgroup analyses and sensitivity analyses will be conducted to assess the robustness of the findings.

Dissemination: The network meta-analysis will provide useful information on antidepressant treatment for child and adolescent depression. The results will be

\section{Strengths and limitations of this study}

- This Bayesian network meta-analysis can integrate direct evidence with indirect evidence from multiple treatment comparisons to estimate the interrelations across all treatments.

- We will comprehensively assess the efficacy, tolerability, acceptability and suicide-related outcomes of first-generation and newer-generation antidepressant medications for depression in children and adolescents.

- Several subgroup and sensitivity analyses will address some clinically relevant questions.

- This method comprehensively synthesises data to provide a clinically useful summary that can guide treatment decisions and guideline development.

disseminated through peer-reviewed publication or conference presentations.

Trial registration number: PROSPERO CRD42015016023.

\section{BACKGROUND}

Depressive disorders in children and adolescents are a major public health problem, demonstrated by the disorders ranking as the third most important in the estimation of disease burden. ${ }^{1}$ The prevalence of experiencing at least one episode of major depression before adulthood is estimated to be approximately $1-2 \%$ for children (6-12 years old), and $2-5 \%$ for adolescents (13-18 years old). ${ }^{2}$ 
The course of major depression in young people is often characterised by frequent recurrence, protracted episodes and comorbid psychiatric disorders. ${ }^{3}$ The consequences of an untreated episode of major depression in young people are likely to be serious impairment in social functioning, for example, poor school achievement, or relational problems with family members and peers. ${ }^{4}$ A report from the American Academy of Child and Adolescent Psychiatry (AACAP) suggested that depression is responsible for over 500000 suicide attempts by children and adolescents a year, with most of this group diagnosed with treatable forms of mental illness. ${ }^{5}{ }^{5}$ Thus, early recognition, diagnosis and treatment of depression in children and adolescents is an important strategy for curbing the rising rate of youth suicide, seen in many developed and advanced developing nations. ${ }^{7}$

Since the late 1960s, first-generation antidepressants, for example, tricyclic antidepressant (TCA) drugs, have been used to treat depressive symptoms in young patients. ${ }^{8}$ In the US, the use of antidepressant medication in children and adolescents grew 3-10-fold between 1987 and $1996 .{ }^{9}$ The efficacy of TCAs has been investigated in 13 randomised placebo-controlled trials, ${ }^{10}$ which showed marginal evidence to support the use of TCAs in the treatment of depression in only adolescents. However, methodological deficiencies in these trials, including small sample sizes and diagnostic heterogeneity, restrict statistical inference and generalisability of the findings. At the same time, cardiovascular effects and overdose-related mortality associated with TCA use have greatly limited their utility in clinical practice. ${ }^{11} 12$ Nevertheless, the TCA nortriptyline is still approved by the Food and Drug Administration (FDA) for the treatment of depression in adolescents and adults. ${ }^{13}$

In recent decades, newer-generation antidepressants, including second-generation antidepressants (eg, selective serotonin reuptake inhibitors (SSRIs)) and thirdgeneration antidepressants (eg, serotonin-norepinephrine reuptake inhibitors (SNRIs)), have been widely used for the treatment of depression in children and adolescents. ${ }^{14}$ The frequency of prescription of SSRIs and SNRIs in children and adolescents has progressively increased. ${ }^{15}$ In European countries, there has been a doubling of SSRI use over a 4-year period. ${ }^{16}$ However, only fluoxetine was approved by the US FDA for treating depression in children and adolescents in January 2003. ${ }^{17}$ In the same year, concerns about the increased risk of suicide and suicide attempts with SSRIs were first raised. ${ }^{18}$ In September 2004, the FDA cautioned practitioners in the use of antidepressant medications in children and adolescents. ${ }^{19}$ Similar warnings were issued by other health regulatory agencies. ${ }^{20-22}$ Thus, concerns about this issue have refocused attention on the question of how effective antidepressant medications are in treating youth depression.

Nonetheless, currently, no published meta-analysis has combined direct and indirect evidence for the use of antidepressant medications on children and adolescents, though it is an important study to perform, given the conflicting results regarding the efficacy and tolerability of various antidepressant medications in this age group, and lack of head-to-head trials of such drugs. ${ }^{23-25}$ For these reasons, we will employ a network meta-analysis-a methodological approach that allows the simultaneous comparison of multiple psychotherapeutic interventions within a single analysis, while preserving randomisation. ${ }^{26}$ This approach will be used to integrate direct evidence (from studies directly comparing interventions) with indirect evidence (information about two treatments derived via a common comparator) from multiple treatment comparisons to estimate the interrelations across all treatments. ${ }^{27}$ We have previously compared the efficacy and acceptability of psychotherapies for depression in children and adolescents, ${ }^{28}$ and the augmentation agents for treatment-resistant depression in adults ${ }^{29}$ in this way. The aim of the network meta-analysis of randomised controlled trials (RCTs) is to systematically reanalyse the efficacy, tolerability, acceptability and suicide risk of both firstgeneration and newer-generation antidepressant medications against active comparator or control conditions, in the treatment of child and adolescent depression.

\section{METHODS}

\section{Criteria for included studies}

\section{Types of studies}

Any prospective RCTs, including cross-over design and cluster randomised trials, will be included. However, quasi-randomised trials (eg, those allocating using alternate days of the week) will be excluded. Trials with sample sizes smaller than 10 will be excluded in this review.

\section{Types of participants}

Children and adolescents (aged from 6 to 18 years when they initially enrolled in the trials) with a primary diagnosis of current major depressive disorder according to standardised diagnostic interviews, for example, the Diagnostic and Statistical Manual of Mental Disorders $(\mathrm{DSM})^{30-32}$ or the International Classification of Diseases (ICD), ${ }^{33} 34$ will be included. Where a trial contains a portion of participants who are over 18 years of age, we will contact the trial authors in order to obtain data for only those participants within our age range. We will exclude trials focusing on child or adolescent bipolar disorder, but will include trials involving patients with comorbid general psychiatric disorders, such as attention deficit hyperactivity disorder, anxiety disorder and substance-related disorder. Also, we will not exclude trials in which participants have a diagnosis of psychotic depression; these participants will be considered within a separate subgroup analysis. However we will exclude trials in which participants have a diagnosis of treatment-resistant depression, because these patients tend to have a different treatment response compared with patients with non-resistant depression. 
Types of interventions

RCTs comparing any first-generation and newergeneration antidepressant drug against active comparator or placebo for treatment of depression in children and adolescents will be included. Trials comparing the same type of antidepressant but at different therapeutic dose (fixed or flexible dose) and different treatment duration will be considered as the same node in the network analysis. We will exclude trials involving combination therapy (ie, combination of antidepressant medications, combination of antidepressant medication with psychotherapy, or other non-psychotherapeutic interventions); however, trials will be considered as eligible if the concomitant psychotherapy is not predefined in the study.

\section{Types of outcome measures}

The acute phase will be defined as from 4 to 16 weeks, and if a trial presents data beyond 16 weeks or for more than one time period within our predefined acute phase periods, we will take the 8-week or close to 8-week time point. ${ }^{35}$ We will exclude trials with treatment duration of $<4$ weeks. Where depression symptoms are measured using more than one depression scale in a trial, we will extract data from the depressive scales on the basis of a hierarchy of rating scales. This hierarchy will be based on psychometric properties and appropriateness for use with children and adolescents, and for consistency of use across trials (referred from the Hetrick ${ }^{14}$ et al study) (table 1). The Children's Depression Rating Scale Revised (CDRS-R) ${ }^{36}$ is adapted for children and adolescents from the Hamilton Depression Rating Scale (HAMD), ${ }^{37}$ a tool validated and commonly used in adult populations. ${ }^{38}$ Both the CDRS-R and HAMD have good reliability and validity. ${ }^{38}$ The Beck Depression Inventory (BDI) ${ }^{39}$ and the Children's Depression Inventory $(\mathrm{CDI})^{40}$ are the most commonly used among depression symptom severity self-rated scales and are ranked second highest in the hierarchy.

1. Overall efficacy

The primary outcome for efficacy will be mean improvement in depressive symptoms, as measured by the mean change score of depression rating scales (self-rated or assessor-rated) from baseline to end point.

The secondary outcome for efficacy will be response in depressive symptoms, as estimated by the proportion of patients who achieved a decrease of a certain percentage (eg, a reduction of $50 \%$ or more) in depression rating score. ${ }^{41}$ When 'response' is not reported, we will use 'remission', if available. Remission will be defined as the proportion of patients who achieved a depression rating score below the published threshold (eg, CDRS-R $\leq 28) .{ }^{41}$

2. Overall tolerability

The tolerability of treatment will be defined as side effect discontinuation in this review, as defined by the
Table 1 Hierarchy of depression symptom severity measurement scales

\begin{tabular}{|c|c|c|}
\hline Hierarchy & $\begin{array}{l}\text { Depression symptom } \\
\text { severity measurement } \\
\text { scales }\end{array}$ & Abbreviation \\
\hline 1 & $\begin{array}{l}\text { Children's Depression Rating } \\
\text { Scale }\end{array}$ & CDRS \\
\hline 2 & $\begin{array}{l}\text { Hamilton Depression Rating } \\
\text { Scale }\end{array}$ & HAMD \\
\hline 3 & $\begin{array}{l}\text { Montgomery Asberg } \\
\text { Depression Rating Scale }\end{array}$ & MADRS \\
\hline 4 & Beck Depression Inventory & BDI \\
\hline 5 & $\begin{array}{l}\text { Children's Depression } \\
\text { Inventory }\end{array}$ & CDI \\
\hline 6 & $\begin{array}{l}\text { Schedule for Affective } \\
\text { Disorders and Schizophrenia } \\
\text { for School Aged Children }\end{array}$ & K-SADS \\
\hline 7 & $\begin{array}{l}\text { Mood and Feeling } \\
\text { Questionnaire }\end{array}$ & MFQ \\
\hline 8 & $\begin{array}{l}\text { Reynolds Adolescent } \\
\text { Depression Scale }\end{array}$ & RADS \\
\hline 9 & Bellevue Index of Depression & BID \\
\hline 10 & Child Depression Scale & CDS \\
\hline 11 & $\begin{array}{l}\text { Centre for Epidemiologic } \\
\text { Studies Depression Scale }\end{array}$ & CESD \\
\hline 12 & Child Assessment Schedule & CAS \\
\hline 13 & $\begin{array}{l}\text { Child Behaviour Checklist- } \\
\text { Depression }\end{array}$ & CBCL-D \\
\hline
\end{tabular}

proportion of patients who discontinued treatment due to adverse events during the study.

3. Overall acceptability

The acceptability of treatment will be defined as allcause discontinuation, as measured by the proportion of patients who discontinued treatment (during the delivery of the intervention) up to the post-intervention time point.

4. Suicide-related outcomes

Suicide-related dichotomous and continuous outcomes will be measured. If data are available, we will extract the number of participants with suicide-related events (combined suicidal ideation and suicidal behaviour) during the acute treatment, as measured on a standardised, validated and reliable rating scale, or reported cases of suicidality. ${ }^{42}$ In addition, we will also collect data on suicidal ideation as a continuous outcome where a standardised, validated and reliable rating scale, such as the Suicidal Ideation Questionnaire-Junior High School version (SIQ-JR) ${ }^{43}$ has been used.

\section{Data sources and search strategy}

Seven electronic databases (PubMed, EMBASE, the Cochrane Library, Web of Science, CINAHL, LiLACS and PsycINFO) will be searched from 1966 to December 2013 (updated to May, 2015), with Medical Subject Headings (MeSH) and text words: 'depress*' or 'dysthymi*' or 'mood disorder*' or 'affective disorder*' and 'selective serotonin reuptake inhibitor*' or 'SSRIs' or 
'serotonin norepinephrine reuptake inhibitor*' or 'SNRIs' or 'noradrenergic and specific serotonergic antidepressants' or 'NaSSA' or 'citalopram' or 'fluoxetine' or 'paroxetine' or 'sertraline' or 'escitalopram [' or 'fluvoxamine' or 'venlafaxine' or 'duloxetine' or 'milnacipran' or 'reboxetine' or 'bupropion' or 'mirtazapine' or 'tricyclic' or 'amersergide' or 'amineptine' or 'amitriptyline' or 'amoxapine' or 'butriptyline' or 'chlorpoxiten' or 'clomipramine' or 'clorimipramine' or 'demexiptiline' or 'desipramine' or 'dibenzipin' or 'dothiepin' or 'doxepin' or 'imipramine' or 'lofepramine' or 'melitracen' or 'metapramine' or 'nortriptyline' or 'noxiptiline' or 'opipramol' or 'protriptyline' or 'quinupramine' or 'tianeptine' or 'trimipramine' and 'adolesc*' or 'child*' or 'boy*' or 'girl*' or 'juvenil*' or 'minors' or 'paediatri*' or 'pediatri*' or 'pubescen*' or 'school*' or 'student*' or 'teen*' or 'young' or 'youth*'. Also, ClinicalTrials.gov, WHO's trial portal, and US FDA reports will be reviewed. There are no restrictions on language or type of publication. Additional studies will be searched in the reference lists of all identified publications including relevant meta-analyses and systematic reviews. All relevant authors and principal manufacturers will be contacted to supplement incomplete reports of the original papers or to provide new data for unpublished trials.

\section{Study selection}

Two reviewers (BQ and YL) will independently scan citations at the title/abstract level identified from the search strategies and then obtain potentially relevant studies in full text, and determine whether to include them by the same eligibility criteria. Besides, the references of relevant reviews and included trials will also be checked by the two reviewers. The reasons for exclusion of trials will be reported in the characteristics of excluded studies tables. Any disagreements will be resolved by a third review author (XZ).

\section{Data extraction and risk of bias assessment}

Two independent reviewers (YL, BQ) will independently extract the key trial parameters using a standardised data abstraction form and assess the risk of bias. The standardised data extraction forms will include the trial characteristics (eg, first listed author, publication year, journal, country, institution and sponsor), patient characteristics (eg, diagnostic criteria for depression, the type of patients, the number of patients, level of depressive symptoms, comorbidities, the age of patients and the gender of patients), intervention details (eg, antidepressant type, dose of antidepressant and the duration of treatment) and outcome measures (efficacy, tolerability, acceptability and suicide-related outcome). The risk of bias in trials will be assessed by the Cochrane risk of bias tool. ${ }^{42}$ Trials attracting a rating of high risk of bias in one or more domains will be considered as 'high risk', low risk of bias in all domains as 'low risk' and one or more unclear risk of bias in each domain as 'unclear risk'. ${ }^{42}$ Any disagreements will be resolved by a third review author (XZ). In addition, we will calculate the inter-rater reliability of the two raters.

\section{Data synthesis and analysis}

We will perform Bayesian network meta-analysis to compare the relative outcomes of different antidepressant medications and placebo with each other from the median of the posterior distribution. ${ }^{26} 27$ The pooled estimates of standardised mean difference (SMD) with 95\% credible intervals (CrIs) will be calculated for the continuous outcomes; and ORs with $95 \%$ CrIs will be calculated for the categorical outcomes. The SMD is that the difference in means (MD) of change scores between the two groups divided by the pooled SD of the measurements, with a negative SMD value, indicates greater symptomatic relief. In the presence of minimally informative priors, CrIs can be interpreted similarly to CIs, and at conventional levels of statistical significance a two sided $\mathrm{p}<0.05$ can be assumed if $95 \%$ CrIs do not include 0 . If means and SDs are not provided, we will calculate them from the $\mathrm{p}$ value or other statistical indices as described elsewhere. ${ }^{44}{ }^{45}$ Results from intention-to-treat analysis (ITT) or modified ITT will be preferred over results from completer analyses, while we will also consider the data set for the means and SDs that are presented in the literature.

The pooled estimates will be obtained using the Markov Chains Monte Carlo method. Two Markov chains will be run simultaneously with different arbitrarily chosen initial values. To ensure convergence, trace plots and the Brooks-Gelman-Rubin statistic will be assessed. ${ }^{46}$ Convergence will be found to be adequate after running 50000 samples for both chains. These samples will be then discarded as 'burn-in', and posterior summaries will be based on 100000 subsequent simulations. The node splitting method will be used to calculate the inconsistency of the model, which separates evidence on a particular comparison into direct and indirect evidence. ${ }^{47}$ Probability values will be summarised and reported as surface under the cumulative ranking curve (SUCRA) and rankograms, a simple transformation of the mean rank used to provide a hierarchy of the treatments and accounts for both the location and the variance of all relative treatment effects. ${ }^{48}$ Network meta-analysis will be performed using the WinBUGS software package (V.1.4.3, MRC Biostatistics Unit, Cambridge, UK) with random-effects models for multiarm trials. The other analyses will be performed and presented by the Stata V.11.0 and R 2.11.1 software packages.

\section{Subgroup analyses}

The antidepressant medications will be coded according to clinical characteristics, risk of bias and sample size. We will conduct the subgroup analyses of data in primary outcome for efficacy. We will perform the following subgroup analyses by using the meta-regression model, and calculate Somer's D (a correlation 
coefficient for a dichotomous and an ordinal variable): ${ }^{49}$ (1) sex ratio (male-to-female ratio $>1$ vs male-to-female ratio $<1$ ); (2) age group; (3) treatment duration; (4) severity of depressive symptom (mild-to-moderate vs moderate-to-severe); (5) comorbid general psychiatric disorders (with vs without comorbidity); (6) risk of bias ('high risk' literature vs 'unclear and low risk' literature); (7) sample size; (8) company sponsor (with vs without sponsor); and (9) the type of trials (published literature vs unpublished literature). When there is the limitation of a small number of comparisons for some potential modifiers in carrying out subgroup analyses on these variables, we will perform the sensitivity analyses by omitting specific trials from the overall analysis.

\section{Other analyses}

Funnel plot analyses will be performed to check for publication bias. Moreover, we will carry out meta-regression analyses to investigate the effect of sponsorship or year published on outcome estimate.

\section{Ethics and dissemination}

This network meta-analysis does not need ethical approval, as data used here are not individual or private. The analysis will be published in a peer-reviewed journal. The results will provide a general overview, and evidence of efficacy and safety of antidepressant medications for depression in children and adolescents. The results will also have implications for clinical practice and further research.

\section{Author affiliations}

${ }^{1}$ Department of Neurology, The First Affiliated Hospital of Chongqing Medical University, Chongqing, China

${ }^{2}$ Research Department of Clinical, Educational and Health Psychology, University College London, London, UK

${ }^{3}$ Department of Child and Adolescent Psychiatry, AP-HP, Hôpital PitiéSalpétrière, Institut des Systèmes Intelligents et de Robotiques (ISIR), Centre National pour la Recherche Scientifique, Université Pierre et Marie Curie, Paris, France

${ }^{4}$ Department of Diagnostic, Clinical, and Public Health Medicine, University of Modena and Reggio, Emilia, Modena, Italy

${ }^{5}$ Department of Psychology, Appalachian State University, Boone, North Carolina, USA

\section{Collaborators Andrea Cipriani and Sarah E Hetrick.}

Contributors $\mathrm{XZ}$ and $\mathrm{BQ}$ conceived the study and drafted the protocol. $\mathrm{XZ}$ and $P X$ wrote the first draft of the manuscript. KDM, CW and DC assisted in protocol design and revision. $\mathrm{YL}$ and $\mathrm{YZ}$ participated in the search strategy development. CDG and $B Q$ participated in the design of data synthesis and analysis. All the authors have approved the publication of the protocol.

Funding This work is supported by the National Basic Research Program of China (973 Program) (grant number 2009CB918300).

Competing interests None declared

Provenance and peer review Not commissioned; externally peer reviewed.

Open Access This is an Open Access article distributed in accordance with the Creative Commons Attribution Non Commercial (CC BY-NC 4.0) license, which permits others to distribute, remix, adapt, build upon this work noncommercially, and license their derivative works on different terms, provided the original work is properly cited and the use is non-commercial. See: http:// creativecommons.org/licenses/by-nc/4.0/

\section{REFERENCES}

1. Ferrari AJ, Charlson FJ, Norman RE, et al. Burden of depressive disorders by country, sex, age, and year: findings from the global burden of disease study 2010. PLoS Med 2013;10:e1001547.

2. Ryan ND. Treatment of depression in children and adolescents. Lancet 2005;366:933-40.

3. Rao U, Ryan ND, Birmaher B, et al. Unipolar depression in adolescents: clinical outcome in adulthood. J Am Acad Child Adolesc Psychiatry 1995;34:566-78.

4. Jaffee SR, Moffitt TE, Caspi A, et al. Differences in early childhood risk factors for juvenile-onset and adult-onset depression. Arch Gen Psychiatry 2002;59:215-22.

5. Office of the Surgeon General. The Surgeon General's call to action to prevent suicide. Washington DC: Department of Health and Human Services, 1999.

6. Centers for Disease Control and Prevention (CDC). Suicide trends among youths and young adults aged 10-24 years-United States, 1990-2004. MMWR Morb Mortal Wkly Rep 2007;56:905-8.

7. Pfeffer CR. Suicide among youth: perspectives on risk and prevention. Washington DC: American Psychiatric Press, 1989 : 203-25.

8. Lieberman JAI. History of the use of antidepressants in primary care. $J$ Clin Psychiatry 2003:5:6-10.

9. Zito JM, Safer DJ, DosReis S, et al. Psychotropic practice patterns for youth: a 10-year perspective. Arch Pediatr Adolesc Med 2003;157:17-25.

10. Hazell P, Mirzaie M. Tricyclic drugs for depression in children and adolescents. Cochrane Database Syst Rev 2013;6:CD002317.

11. Biederman J. Sudden death in children treated with a tricyclic antidepressant. J Am Acad Child Adolesc Psychiatry 1991:30:495-8.

12. Varley CK, McClellan J. Case study: two additional sudden deaths with tricyclic antidepressants. J Am Acad Child Adolesc Psychiatry 1997;36:390-4.

13. Green WH. Child and Adolescent clinical psychopharmacology. 3rd edn. Philadelphia: Lippincott Williams and Wilkins, 2003:169-72.

14. Hetrick SE, McKenzie JE, Cox GR, et al. Newer generation antidepressants for depressive disorders in children and adolescents. Cochrane Database Syst Rev 2012;11:CD004851.

15. Bennett K, Teeling M, Feely J. Overprescribing antidepressants to children: pharmacoepidemiological study in primary care. BMJ 2005;331:1451-2

16. Fegert JM, Kölch M, Zito JM, et al. Antidepressant use in children and adolescents in Germany. J Child Adolesc Psychopharmacol 2006;16:197-206.

17. Gentile S. Antidepressant use in children and adolescents diagnosed with major depressive disorder: what can we learn from published data? Rev Recent Clin Trials 2010;5:63-75.

18. Healy D. Lines of evidence on the risks of suicide with selective serotonin reuptake inhibitors. Psychother Psychosom 2003;72:71-9.

19. U.S. Food and Drug Administration (FDA). Suicidality in children and adolescents being treated with antidepressant medications. http:// www.fda.gov/drugs/drugsafety/postmarketdrugsafetyinformation forpatientsandproviders/ucm161679.htm (accessed 11 Dec 2014).

20. Adverse Drug Reactions Advisory Committee. Use of SSRI antidepressants in children and adolescents. https://www.tga.gov.au/ use-ssri-antidepressants-children-and-adolescents-june-2004 (accessed 1 Dec 2014)

21. Canadian Psychiatric Association Bulletin. Health Canada to examine SSRIs in children, April 2004. http://ww1.cpa-apc.org/ Publications/Archives/Bulletin/2004/april/news32En.asp (accessed 18 Dec 2014)

22. National Institute of Mental Health. Antidepressant medications for children and adolescents: information for parents and caregivers. http://www.nimh.nih.gov/health/topics/child-and-adolescent-mentalhealth/antidepressant-medications-for-children-and-adolescentsinformation-for-parents-and-caregivers.shtml (accessed 14 Dec 2014)

23. Papanikolaou K, Richardson C, Pehlivanidis A, et al. Efficacy of antidepressants in child and adolescent depression: a meta-analytic study. J Neural Transm 2006;113:399-415.

24. Whittington CJ, Kendall T, Fonagy $P$, et al. Selective serotonin reuptake inhibitors in childhood depression: systematic review of published versus unpublished data. Lancet 2004;363: 1341-5.

25. Qin B, Zhang $Y$, Zhou $X$, et al. Selective serotonin reuptake inhibitors versus tricyclic antidepressants in young patients: a meta-analysis of efficacy and acceptability. Clin Ther 2014;36: 1087-95.

26. Salanti G, Higgins JP, Ades AE, et al. Evaluation of networks of randomized trials. Stat Methods Med Res 2008;17:279-301. 
27. Lu G, Ades AE. Combination of direct and indirect evidence in mixed treatment comparisons. Stat Med 2004;23:3105-24.

28. Zhou X, Ravindran A, Qin B, et al. Comparative efficacy, acceptability and tolerability of augmentation agents in treatment-resistant depression: systematic review and network meta-analysis. J Clin Psychiatry 2015;76:e487-98.

29. Qin B, Zhou X, Michael KD, et al. Psychotherapy for depression in children and adolescents: study protocol for a systematic review and network meta-analysis. BMJ Open 2015;5:e005918.

30. American Psychiatric Association. Diagnostic and Statistical Manual of Mental Disorders (DSM-III). 3rd edn. Washington DC: American Psychiatric Association, 1980.

31. American Psychiatric Association. Diagnostic and Statistical Manual of Mental Disorders (DSM-III-R). 3rd edn. Washington DC: American Psychiatric Association, 1987.

32. American Psychiatric Association. Diagnostic and Statistical Manual of Mental Disorders. 4th edn. Washington DC: American Psychiatric Association, 1994.

33. World Health Organization (WHO). The Ninth Revision of the International Classification of Diseases and Related Health Problems (ICD-9). Geneva: World Health Organization, 1978.

34. World Health Organization (WHO). The Tenth Revision of the International Classification of Diseases and Related Health Problems (ICD-10). Geneva: World Health Organization, 1992.

35. Cipriani A, Furukawa TA, Salanti G, et al. Comparative efficacy and acceptability of 12 new-generation antidepressants: a multiple-treatments meta-analysis. Lancet 2009;373:746-58.

36. Poznanski EO, Mokros HB. Children's depression rating scale, revised (CDRS-R): manual. Los Angeles, CA: Western Psychological Services, 1996.

37. Hamilton M. A rating scale for depression. J Neurol Neurosurg Psychiatry 1960;23:56-62.
38. Brooks SJ, Kutcher S. Diagnosis and measurement of depression: a review of commonly utilized instruments. $J$ Child Adolesc Psychopharmacol 2001;11:341-76.

39. Beck AT, Steer R. Beck depression inventory: manual. San Antonio, TX: Psychological Corporation, 1987.

40. Kovacs M. The Children's Depression, Inventory (CDI). Psychopharmacol Bull 1985;21:995-8.

41. Riedel M, Möller HJ, Obermeier M, et al. Response and remission criteria in major depression-a validation of current practice. J Psychiatr Res 2010;44:1063-8.

42. Hammad TA. Review and evaluation of clinical data. Washington DC: US Food and Drug Administration. http://www.fda.gov/ohrms/ dockets/ac/04/briefing/2004-4065b1-10-tab08-hammads-review.pdf (accessed 9 Dec 2014)

43. Reynolds WM. Suicidal ideation questionnaire-junior. Odessa, FL: Psychological Assessment Resources, 1987.

44. Higgins JPT, Green S. Cochrane handbook for systematic reviews of interventions version 5.1. O [updated March 2011]. The Cochrane Collaboration, 2011

45. Follmann D, Elliott $\mathrm{P}$, Suh I, et al. Variance imputation for overviews of clinical trials with continuous response. J Clin Epidemiol 1992;45:769-73.

46. Brooks SP, Gelman A. General methods for monitoring convergence of iterative simulations. J Comput Graph Stat 1998;7:434-55.

47. Lu GB, Ades AE. Assessing evidence inconsistency in mixed treatment comparisons. J Am Stat Assoc 2006;101:447-59.

48. Salanti G, Ades AE, loannidis JP. Graphical methods and numerical summaries for presenting results from multiple-treatment metaanalysis: an overview and tutorial. J Clin Epidemiol 2011;64:163-71.

49. Dias S, Sutton AJ, Welton NJ, et al. Evidence synthesis for decision making 3: heterogeneity-subgroups, meta-regression, bias, and bias-adjustment. Med Decis Making 2013;33:618-40. 